\title{
NUMERICAL STUDY OF RAYLEIGH-BENARD PROBLEM UNDER THE EFFECT OF MAGNETIC FIELD
}

\author{
ABDELFATAH ABASHER ${ }^{1, *}$, MOHAMMED GUBARA ${ }^{2}$, SULIMAN SHEEN $^{2}$, \\ IBRAHIM BASHIR ${ }^{2}$ \\ ${ }^{1}$ Mathematics Department, Faculty of Science, Jazan University, Jazan, KSA \\ ${ }^{2}$ Mathematics Department, Faculty of Mathematical Science and Statistic, Al-Neelain University, \\ Khartoum, Sudan
}

${ }^{*}$ Corresponding author: amoaf84@gmail.com

\begin{abstract}
In this paper, a linear stability analysis is studied for Rayliegh-Benard problem with the effect of magnetic field, a perturbation equations is solved numerically by using spectral Chebyshev tau method, the boundaries are considered both are free, both are rigid, the lower is free and the upper is rigid, the results were illustrated graphically and compared with previous studies.
\end{abstract}

\section{InTRODUCTION}

The aim of this work is to solve the perturbation equations that represent Rayleigh-Benard problem under the effect of magnetic field ( [1], Page $(160,161))$ as follows

$$
\begin{gathered}
\frac{\partial \mathbf{u}}{\partial t}=-\nabla\left(\frac{\delta p}{\rho_{0}}+\mu \frac{\mathbf{H} \cdot \mathbf{h}}{4 \pi \rho_{0}}\right)+g \alpha \theta \underline{k}+v \nabla^{2} \mathbf{u}+\frac{\mu}{4 \pi \rho_{0}}(\mathbf{H} \cdot \nabla) \mathbf{h}, \\
\frac{\partial \mathbf{h}}{\partial t}=(\mathbf{H} \cdot \nabla) \mathbf{u}+\eta \nabla^{2} \mathbf{h},
\end{gathered}
$$

Received February 24 ${ }^{\text {th }}, 2021$; accepted March 26 ${ }^{\text {th }}$, 2021; published April 28 ${ }^{\text {th }}, 2021$.

2010 Mathematics Subject Classification. 00A69.

Key words and phrases. Rayleigh-Benard problem; linear stability analysis; Chebyshev Tau method.

(C)2021 Authors retain the copyrights of their papers, and all open access articles are distributed under the terms of the Creative Commons Attribution License. 


$$
\frac{\partial \theta}{\partial t}=\beta w+\kappa \nabla^{2} \theta
$$

$$
\nabla \cdot \mathbf{u}=0
$$

where, $\mathbf{u}, \delta p, \theta$ and $\mathbf{h}$ are the perturbation in the velocity, pressure, temperature and magnetic field respectively, $\rho_{0}$ is the density at mean temperature $T_{0}, v$ is the kinematic viscosity, $\kappa$ is the thermal diffusivity, $\alpha$ the coefficient of volume expansion, $\mu$ is the magnetic permeability, $\eta$ is the resistivity and $\beta$ is the temperature gradient defined as

$$
\beta=\frac{T_{0}-T_{d}}{d}
$$

The problem is studied analytically by various authors ( [1]- [4], [12]- [14]). Also many numerical methods were used, one of these method is Galerkin method which discussed in [14]. In this paper we used the numerical method namely Spectral Chebyshev tau method to determine the conditions of instability for various cases of the boundary conditions. The paper outlined as follows.

In section 1 we formulate the governing mathematical perturbation equations. In section 2 a linear stability analysis for the perturbation equations. In section 3 we describe the method of solution Spectral Chebyshev Tau method for the three cases of the boundary conditions. Finally, we present our numerical results in which are computed using MATLAB.

\section{Linear Stability analysis}

By taking the curl operator of equation (1.1), we get

$$
\frac{\partial \omega}{\partial t}=g \alpha\left(\frac{\partial \theta}{\partial y} \underline{i}-\frac{\partial \theta}{\partial x} j\right)+v \nabla^{2} \omega+\frac{\mu}{4 \pi \rho_{0}}(\mathbf{H} \cdot \nabla) \mathbf{v}
$$

where $\omega=\nabla \times \mathbf{u}$ is the vorticity vector and $\mathbf{v}=\nabla \times \mathbf{h}$ is the current density induced by the perturbation.

Taking the curl operator of (2.1) again, we get

$$
\frac{\partial \nabla^{2} \mathbf{u}}{\partial t}=-g \alpha\left(\frac{\partial^{2} \theta}{\partial z \partial x} \underline{i}+\frac{\partial^{2} \theta}{\partial z \partial y} \underline{j}-\left(\frac{\partial^{2} \theta}{\partial x^{2}}+\frac{\partial^{2} \theta}{\partial y^{2}}\right) \underline{k}\right)+v \nabla^{4} \mathbf{u}+\frac{\mu}{4 \pi \rho_{0}}(\mathbf{H} \cdot \nabla) \nabla^{2} \mathbf{h} .
$$

Take the curl of equation (1.2), we get

$$
\frac{\partial \mathbf{v}}{\partial t}=(\mathbf{H} \cdot \nabla) \omega+\eta \nabla^{2} \mathbf{v}
$$


Now by equating the $z$-component of equation (1.2), (2.1), (2.2) and (2.3) respectively, we get

$$
\begin{gathered}
\frac{\partial \zeta}{\partial t}=v \nabla^{2} \zeta+\frac{\mu}{4 \pi \rho_{0}}(\mathbf{H} \cdot \nabla) \xi \\
\frac{\partial \nabla^{2} w}{\partial t}=g \alpha\left(\frac{\partial^{2} \theta}{\partial x^{2}}+\frac{\partial^{2} \theta}{\partial y^{2}}\right)+v \nabla^{4} w+\frac{\mu}{4 \pi \rho_{0}}(\mathbf{H} \cdot \nabla) h_{z}, \\
\frac{\partial \xi}{\partial t}=\eta \nabla^{2} \xi+(\mathbf{H} \cdot \nabla) \zeta,
\end{gathered}
$$

where $w, \zeta$ and $\xi$ and $h_{z}$ are the $z$-components of the velocity, the vorticity, the current density and the magnetic field respectively. When the direction of the magnetic field coincides with the vertical direction $\mathbf{H}=(0,0, H)$, the required perturbation equations become

$$
\begin{gathered}
\frac{\partial}{\partial t} \nabla^{2} w=g \alpha\left(\frac{\partial^{2} \theta}{\partial x^{2}}+\frac{\partial^{2} \theta}{\partial y^{2}}\right)+v \nabla^{4} w+\frac{\mu H}{4 \pi \rho_{0}} \frac{\partial}{\partial z} \nabla^{2} h_{z} \\
\frac{\partial h_{z}}{\partial t}=\eta \nabla^{2} h_{z}+H \frac{\partial w}{\partial z} \\
\frac{\partial \xi}{\partial t}=\eta \nabla^{2} \xi+H \frac{\partial \zeta}{\partial z} \\
\frac{\partial \zeta}{\partial t}=v \nabla^{2} \zeta+\frac{\mu H}{4 \pi \rho_{0}} \frac{\partial \xi}{\partial z}
\end{gathered}
$$

In addition to equation (1.4).

The boundary conditions of $\theta, w$ and $\zeta$ for the three cases free-free, rigid-rigid and rigid-free are

$$
\left\{\begin{array}{l}
\theta=0, w=0 \quad \text { at } z=0 \text { and } z=d \\
\frac{d \zeta}{d z}=0, \frac{d^{2} w}{d z^{2}}=0 \quad \text { at free boundary } \\
\zeta=0, \frac{d w}{d z}=0 \quad \text { at rigid boundary. }
\end{array}\right.
$$




\section{Normal Mode Analysis}

Let $w, \theta, \zeta, h_{z}$ and $\xi$ be defined as

$$
\left(\begin{array}{c}
z \theta \\
\zeta \\
w \\
\xi \\
h_{z}
\end{array}\right)=\left(\begin{array}{c}
\Theta(z) \\
Z(z) \\
W(z) \\
X(z) \\
K(z)
\end{array}\right) \exp \left(i\left(k_{x} x+k_{y} y\right)+\gamma t\right)
$$

where $k=\sqrt{k_{x}^{2}+k_{y}^{2}}$ is the wave number and $\gamma$ is a constant. Substitute (3.1) into the system (1.4), (2.8)-(2.11), the system become

$$
\begin{gathered}
\gamma \Theta=\beta W+\kappa\left(\frac{d^{2}}{d z^{2}}-k^{2}\right) \Theta \\
\gamma K=\eta\left(\frac{d^{2}}{d z^{2}}-k^{2}\right) K+H \frac{d W}{d z}, \\
\gamma\left(\frac{d^{2}}{d z^{2}}-k^{2}\right) W=-g \alpha k^{2} \Theta+v\left(\frac{d^{2}}{d z^{2}}-k^{2}\right)^{2} W+\frac{\mu H}{4 \pi \rho_{0}} \frac{d}{d z}\left(\frac{d^{2}}{d z^{2}}-k^{2}\right) K \\
\gamma X=\eta\left(\frac{d^{2}}{d z^{2}}-k^{2}\right) X+H \frac{d Z}{d z} \\
\gamma Z=v\left(\frac{d^{2}}{d z^{2}}-k^{2}\right) Z+\frac{\mu H}{4 \pi \rho_{0}} \frac{d X}{d z} .
\end{gathered}
$$

And the boundary conditions (2.12) become

$$
\left\{\begin{array}{l}
\Theta=0, W=0 \quad \text { at } z=0 \text { and } z=d \\
\frac{d Z}{d z}=0, \frac{d^{2} W}{d z^{2}}=0 \quad \text { at free boundary } \\
Z=0, \frac{d W}{d z}=0 \quad \text { at rigid boundary. }
\end{array}\right.
$$

Define the following non-dimensional variables,

$$
a=k d, \quad \sigma=\frac{\gamma d^{2}}{v}, \quad z^{*}=\frac{z}{d}, \quad P_{1}=\frac{v}{\kappa}, \quad \text { and } \quad P_{2}=\frac{v}{\eta},
$$

the operators $\frac{d}{d z}=\frac{1}{d} \frac{d}{d z^{*}}$ and $\frac{d^{2}}{d z^{2}}=\frac{1}{d^{2}} \frac{d^{2}}{d z^{* 2}}$, assume $D=\frac{d}{d z^{*}}$, then by substituting (3.8) into the system (3.2)-(3.6), we get

$$
\left(D^{2}-a^{2}-P_{1} \sigma\right) \Theta=-\left(\frac{\beta d^{2}}{\kappa}\right) W
$$




$$
\begin{gathered}
\left(D^{2}-a^{2}-P_{2} \sigma\right) K=-\left(\frac{H d}{\eta}\right) D W \\
\left(D^{2}-a^{2}\right)\left(D^{2}-a^{2}-\sigma\right) W+\left(\frac{\mu H d}{4 \pi \rho_{0} v}\right) D\left(D^{2}-a^{2}\right) K=\left(\frac{g \alpha d^{2}}{v}\right) a^{2} \Theta, \\
\left(D^{2}-a^{2}-P_{2} \sigma\right) X=-\left(\frac{H d}{\eta}\right) D Z \\
\left(D^{2}-a^{2}-\sigma\right) Z=-\left(\frac{\mu H d}{4 \pi \rho_{0} v}\right) D X
\end{gathered}
$$

At the marginal state $(\sigma=0)$, then equations (3.9)-(3.13) become

$$
\begin{gathered}
\left(D^{2}-a^{2}\right) \Theta=-\left(\frac{\beta d^{2}}{\kappa}\right) W, \\
\left(D^{2}-a^{2}\right) K=-\left(\frac{H d}{\eta}\right) D W, \\
\left(D^{2}-a^{2}\right)^{2} W+\left(\frac{\mu H d}{4 \pi \rho_{0} v}\right) D\left(D^{2}-a^{2}\right) K=\left(\frac{g \alpha d^{2}}{v}\right) a^{2} \Theta \\
\left(D^{2}-a^{2}\right) X=-\left(\frac{H d}{\eta}\right) D Z, \\
\left(D^{2}-a^{2}\right) Z=-\left(\frac{\mu H d}{4 \pi \rho_{0} v}\right) D X,
\end{gathered}
$$

and the boundary conditions (3.7) become

$$
\left\{\begin{array}{l}
\Theta=0, \quad W=0 \quad \text { at } z=0 \text { and } z=1 \\
D Z=0, \quad D^{2} W=0 \quad \text { at free boundary } \\
Z=0, \quad D W=0 \quad \text { at rigid boundary. }
\end{array}\right.
$$

By Taking the operator $D$ for equation (3.15) and substituting in (3.16), we get

$$
\left(D^{2}-a^{2}\right)^{2} W-Q D^{2} W=\left(\frac{g \alpha d^{2}}{v}\right) a^{2} \Theta,
$$


where,

$$
Q=\frac{\mu H^{2} d^{2}}{4 \pi \rho_{0} v \eta}
$$

is Chandrasekhar number [1]. Taking the operator $\left(D^{2}-a^{2}\right)$ for equation (3.20), and using equation (3.14), we get

$$
\left(D^{2}-a^{2}\right)\left[\left(D^{2}-a^{2}\right)^{2}-Q D^{2}\right] W=-R a^{2} W
$$

where,

$$
R=\frac{g \alpha \beta d^{4}}{\kappa v}
$$

is Rayleigh number.

By Taking the operator $\left(D^{2}-a^{2}\right)$ for equation (3.18) and using (3.17), we get

$$
\left[\left(D^{2}-a^{2}\right)^{2}-Q D^{2}\right] Z=0
$$

we must seek the solution of (3.22) and (3.24) subject to the boundary conditions (3.19).

\section{Spectral Chebyshev tau Method}

Spectral Chebyshev tau method is a numerical method to solve the differential equations and the eigen values problems, (see [6], [7], [8] and [10]).

To solve equations (3.22) and (3.24) subject to the boundary conditions (3.19), first convert the domain to Chebyshev polynomials domain $[-1,1]$, use the relation $x=2 z-1$, if $z \in[0,1]$ implies $x \in[-1,1]$ and the derivative $\frac{d}{d z}=2 \frac{d}{d x}, \frac{d^{2}}{d z^{2}}=4 \frac{d^{2}}{d x^{2}}$, then (3.22), (3.24) and (3.19) become

$$
\left(4 D^{2}-a^{2}\right)\left[\left(4 D^{2}-a^{2}\right)^{2}-4 Q D^{2}\right] W=-R a^{2} W
$$

$$
\begin{gathered}
{\left[\left(4 D^{2}-a^{2}\right)^{2}-4 Q D^{2}\right] Z=0,} \\
\left\{\begin{array}{l}
W=0 \quad \text { at } x=-1 \text { and } x=1, \\
D Z=0, \quad D^{2} W=0 \quad \text { at free boundary } \\
Z=0, \quad D W=0 \quad \text { at rigid boundary }
\end{array}\right.
\end{gathered}
$$

where $D=\frac{d}{d x}$.

Let $S=\left[\left(4 D^{2}-a^{2}\right)^{2}-4 Q D^{2}\right] W$ then we can write (4.1) - (4.3) as

$$
\left[\left(4 D^{2}-a^{2}\right)^{2}-4 Q D^{2}\right] W-S=0
$$




$$
\begin{gathered}
\left(4 D^{2}-a^{2}\right) S=-R a^{2} W \\
{\left[\left(4 D^{2}-a^{2}\right)^{2}-4 Q D^{2}\right] Z=0,} \\
\left\{\begin{array}{l}
W=0, S=0 \quad \text { at } x=-1 \text { and } x=1, \\
D Z=0, \quad D^{2} W=0 \quad \text { at free boundary } \\
Z=0, \quad D W=0 \quad \text { at rigid boundary. }
\end{array}\right.
\end{gathered}
$$

Now, expand $W, S$ and $Z$ as Chebyshev polynomials

$$
\begin{gathered}
W=\sum_{n=0}^{N} w_{n} T_{n}(x)=\left[\begin{array}{lll}
w_{0} & \cdots & w_{N}
\end{array}\right]\left[\begin{array}{c}
T_{0} \\
\vdots \\
T_{N}
\end{array}\right]=\mathbf{W} \phi, \\
S=\sum_{n=0}^{N} s_{n} T_{n}(x)=\left[\begin{array}{lll}
s_{0} & \cdots & s_{N}
\end{array}\right]\left[\begin{array}{c}
T_{0} \\
\vdots \\
T_{N}
\end{array}\right]=\mathbf{S} \phi, \\
Z=\sum_{n=0}^{N} z_{n} T_{n}(x)=\left[\begin{array}{lll}
z_{0} & \cdots & z_{N}
\end{array}\right]\left[\begin{array}{c}
T_{0} \\
\vdots \\
T_{N}
\end{array}\right]=\mathbf{Z} \phi,
\end{gathered}
$$

where $\mathrm{W}, \mathrm{S}$ and $\mathrm{Z}$ are row vectors represent the coefficients of $W, S$ and $Z$ respectively, and $\phi$ is the vector of chebyshev polynomials $T_{0}$ up to $T_{N}$. Furthermore we can expand the derivatives $D W, D^{2} W$ and $D^{4} W$ as chebyshev polynomials as

$$
\begin{gathered}
D W=\sum_{n=0}^{N} w_{n}^{(1)} T_{n}(x)=\mathbf{W} \mathbf{D} \phi, \\
D^{2} W=\sum_{n=0}^{N} w_{n}^{(2)} T_{n}(x)=\mathbf{W D}^{2} \phi,
\end{gathered}
$$

$$
D^{4} W=\sum_{n=0}^{N} w_{n}^{(4)} T_{n}(x)=\mathbf{W D}^{4} \phi,
$$


similarly for the derivatives of $S$ and $Z$

$$
\begin{aligned}
& D^{2} S=\sum_{n=0}^{N} s_{n}^{(2)} T_{n}(x)=\mathbf{S D}^{2} \phi, \\
& D^{2} Z=\sum_{n=0}^{N} z_{n}^{(2)} T_{n}(x)=\mathbf{Z D}^{2} \phi,
\end{aligned}
$$

Where $\mathbf{D}$ and $\mathbf{D}^{2}$ are $(N+1) \times(N+1)$ matrices represent the coefficients of the first and second chebyshev derivatives, (see [6] and [10] for more details of formulations of $\mathbf{D}$ and $\mathbf{D}^{2}$ ).

$$
\begin{aligned}
& \mathbf{D}=\left(\begin{array}{ccccccc}
0 & 0 & 0 & 0 & 0 & \cdots & 0 \\
1 & 0 & 0 & 0 & 0 & \cdots & 0 \\
0 & 4 & 0 & 0 & 0 & \cdots & 0 \\
3 & 0 & 6 & 0 & 0 & \cdots & 0 \\
0 & 8 & 0 & 8 & 0 & \cdots & 0 \\
\vdots & \vdots & \vdots & \vdots & \vdots & \ddots & 0 \\
N & 0 & 2 N & 0 & 2 N & \cdots & 0
\end{array}\right) \\
& \mathbf{D}^{2}=\left(\begin{array}{cccccccc}
0 & 0 & 0 & 0 & 0 & 0 & \cdots & 0 \\
0 & 0 & 0 & 0 & 0 & 0 & \cdots & 0 \\
4 & 0 & 0 & 0 & 0 & 0 & \cdots & 0 \\
0 & 24 & 0 & 0 & 0 & 0 & \cdots & 0 \\
32 & 0 & 48 & 0 & 0 & 0 & \cdots & 0 \\
0 & 120 & 0 & 80 & 0 & 0 & \cdots & 0 \\
\vdots & \vdots & \vdots & \vdots & \vdots & \vdots & \ddots & 0 \\
0 & N\left(N^{2}-1\right) & 0 & N\left(N^{2}-9\right) & 0 & N\left(N^{2}-25\right) & \cdots & 0
\end{array}\right),
\end{aligned}
$$

and $\mathbf{D}^{4}=(\mathbf{D})^{4}=\mathbf{D}^{2} \mathbf{D}^{2}$. By substitute (4.8)-(4.15) into equations (4.4)-(4.6) we get,

$$
\begin{gathered}
\mathbf{W}\left[16 \mathbf{D}^{4}-\left(8 a^{2}+4 Q\right) \mathbf{D}^{2}+a^{4} \mathbf{I}\right] \phi-\mathbf{S} \phi=0, \\
\mathbf{S}\left[4 \mathbf{D}^{2}-a^{2} \mathbf{I}\right] \phi=-R a^{2} \mathbf{W} \phi,
\end{gathered}
$$

$$
\mathbf{Z}\left[16 \mathbf{D}^{4}-\left(8 a^{2}+4 Q\right) \mathbf{D}^{2}+a^{4} \mathbf{I}\right] \phi=0
$$


where $\mathbf{I}$ is the identity matrix of order $(N+1)$. By taking the inner product with $T_{n}, n=0,1, . ., N$, for each equation in the system (4.18) - (4.20) and using the property of orthogonality of Chebyshev polynomials, we obtain $3(N+1)$ equations as follows

$$
\begin{gathered}
4 s_{n}^{(2)}-a^{2} s_{n}=-R a^{2} w_{n}, \quad n=0,1, \ldots, N \\
16 z_{n}^{(4)}-\left(8 a^{2}+4 Q\right) z_{n}^{(2)}+a^{4} z_{n}=0 . \quad n=0,1, \ldots, N
\end{gathered}
$$

Rewriting these equations in matrices form as

$$
\mathbf{W}\left[16 \mathbf{D}^{4}-\left(8 a^{2}+4 Q\right) \mathbf{D}^{2}+a^{4} \mathbf{I}\right]-\mathbf{S}=0
$$

$$
\mathbf{Z}\left[16 \mathbf{D}^{4}-\left(8 a^{2}+4 Q\right) \mathbf{D}^{2}+a^{4} \mathbf{I}\right]=0
$$

or,

$$
\mathbf{A X}=R \mathbf{B X}
$$

where $\mathbf{A}$ and $\mathbf{B}$ are square matrices of order $3(N+1)$ given as

$$
\mathbf{A}=\left(\begin{array}{ccc}
\mathbf{L}_{1} & -\mathbf{I} & \mathbf{0} \\
\mathbf{0} & \mathbf{L}_{2} & \mathbf{0} \\
\mathbf{0} & \mathbf{0} & \mathbf{L}_{1}
\end{array}\right), \mathbf{B}=\left(\begin{array}{ccc}
\mathbf{0} & \mathbf{0} & \mathbf{0} \\
-a^{2} \mathbf{I} & \mathbf{0} & \mathbf{0} \\
\mathbf{0} & \mathbf{0} & \mathbf{0}
\end{array}\right) \text {, and } \mathbf{X}=\left(\begin{array}{c}
\mathbf{W}^{T} \\
\mathbf{S}^{T} \\
\mathbf{Z}^{T}
\end{array}\right)
$$

where $\mathbf{L}_{1}=\left[16 \mathbf{D}^{4}-\left(8 a^{2}+4 Q\right) \mathbf{D}^{2}+a^{4} \mathbf{I}\right]^{T}$ and $\mathbf{L}_{2}=\left[4 \mathbf{D}^{2}-a^{2} \mathbf{I}\right]^{T}$. Now returning to the boundary conditions (4.7), the $p$-derivative of Chebyshev polynomials at $x= \pm 1$ is given by the formula

$$
\left.\frac{d^{p} T_{n}}{d x^{p}}\right|_{x= \pm 1}=( \pm 1)^{n+p} \prod_{k=0}^{p-1} \frac{n^{2}-k^{2}}{2 k+1},
$$

For Free-Free boundaries, We have eight boundary conditions as

$$
W=0, \quad D^{2} W=0, \quad S=0 \quad \text { and } \quad D Z=0 \quad \text { for } x= \pm 1 .
$$




$$
\begin{aligned}
& \mathrm{BC} 1: W(-1)=0 \Rightarrow \sum_{n=0}^{N} w_{n} T_{n}(-1)=0 \Rightarrow \sum_{n=0}^{N}(-1)^{n} w_{n}=0, \\
& \mathrm{BC} 2: W(1)=0 \Rightarrow \sum_{n=0}^{N} w_{n} T_{n}(1)=0 \Rightarrow \sum_{n=0}^{N} w_{n}=0 \\
& \mathrm{BC} 3: D^{2} W(-1)=0 \Rightarrow \sum_{n=0}^{N} w_{n} T_{n}^{\prime \prime}(-1)=0 \Rightarrow \sum_{n=0}^{N}(-1)^{n+2} \frac{n^{2}\left(n^{2}-1\right)}{3} w_{n}=0, \\
& \mathrm{BC} 4: D^{2} W(1)=0 \Rightarrow \sum_{n=0}^{N} w_{n} T_{n}^{\prime \prime}(1)=0 \Rightarrow \sum_{n=0}^{N} \frac{n^{2}\left(n^{2}-1\right)}{3} w_{n}=0, \\
& \mathrm{BC} 5: S(-1)=0 \Rightarrow \sum_{n=0}^{N} s_{n} T_{n}(-1)=0 \Rightarrow \sum_{n=0}^{N}(-1)^{n} s_{n}=0 \\
& \mathrm{BC} 6: S(1)=0 \Rightarrow \sum_{n=0}^{N} s_{n} T_{n}(1)=0 \Rightarrow \sum_{n=0}^{N} s_{n}=0 \\
& \mathrm{BC} 7: D Z(-1)=0 \Rightarrow \sum_{n=0}^{N} z_{n} T_{n}^{\prime}(-1)=0 \Rightarrow \sum_{n=0}^{N}(-1)^{n+1} n^{2} z_{n}=0, \\
& \mathrm{BC} 8: D Z(1)=0 \Rightarrow \sum_{n=0}^{N} z_{n} T_{n}^{\prime}(1)=0 \Rightarrow \sum_{n=0}^{N} n^{2} z_{n}=0
\end{aligned}
$$

similarly, for rigid-rigid boundaries,

$$
W=0, \quad D W=0, \quad S=0 \quad \text { and } \quad Z=0 \quad \text { for } x= \pm 1 .
$$

and for rigid-free boundaries,

$$
\begin{aligned}
& W=0, \quad D W=0, \quad S=0 \quad \text { and } \quad Z=0 \quad \text { for } x=-1 \text { rigid. } \\
& W=0, \quad D^{2} W=0, \quad S=0 \quad \text { and } \quad D Z=0 \quad \text { for } x=1 \text { free. }
\end{aligned}
$$

For each case of the boundary conditions, insert $B C 1$ up to $B C 4$ into the rows $(N-2)^{t h}$ up to $(N+1)^{t h}$ of the first column in the matrix $\mathbf{A}$ in (4.27), BC5 and $B C 6$ into the rows $(2 N+1)^{t h}$ and $(2 N+2)^{t h}$ of the second column in $\mathbf{A}, B C 7$ and $B C 8$ into the rows $(3 N+2)^{t h}$ and $(3 N+3)^{t h}$ of the third column in $\mathbf{A}$. The 
corresponding rows in the matrix $\mathbf{B}$ are zeros, then we can write the system (4.27) as follows

$$
\left(\begin{array}{ccc}
\overline{\mathbf{L}}_{1} & -\overline{\mathbf{I}} & \overline{\mathbf{0}} \\
\mathrm{BC} 1 & 0 \ldots 0 & 0 \ldots 0 \\
\mathrm{BC} 2 & 0 \ldots 0 & 0 \ldots 0 \\
\mathrm{BC} 3 & 0 \ldots 0 & 0 \ldots 0 \\
\mathrm{BC} 4 & 0 \ldots 0 & 0 \ldots 0 \\
\overline{\mathbf{0}} & \overline{\mathbf{L}}_{2} & \overline{\mathbf{0}} \\
0 \ldots 0 & \mathrm{BC} 5 & 0 \ldots 0 \\
0 \ldots 0 & \mathrm{BC} 6 & 0 \ldots 0 \\
\overline{\mathbf{0}} & \overline{\mathbf{0}} & \overline{\mathbf{L}}_{1} \\
0 \ldots 0 & 0 \ldots 0 & \mathrm{BC} 7 \\
0 \ldots 0 & 0 \ldots 0 & \mathrm{BC} 8
\end{array}\right)\left(\begin{array}{c}
w_{0} \\
\vdots \\
w_{n} \\
s_{0} \\
\vdots \\
s_{n} \\
z_{0} \\
\vdots \\
z_{n}
\end{array}\right)=R\left(\begin{array}{ccc}
\overline{\mathbf{0}} & \overline{\mathbf{0}} & \overline{\mathbf{0}} \\
0 \ldots 0 & 0 \ldots 0 & 0 \ldots 0 \\
0 \ldots 0 & 0 \ldots 0 & 0 \ldots 0 \\
0 \ldots 0 & 0 \ldots 0 & 0 \ldots 0 \\
0 \ldots 0 & 0 \ldots 0 & 0 \ldots 0 \\
-a^{2} \overline{\mathbf{I}} & \overline{\mathbf{0}} & \overline{\mathbf{0}} \\
0 \ldots 0 & 0 \ldots 0 & 0 \ldots 0 \\
0 \ldots 0 & 0 \ldots 0 & 0 \ldots 0 \\
\overline{\mathbf{0}} & \overline{\mathbf{0}} & \overline{\mathbf{0}} \\
0 \ldots 0 & 0 \ldots 0 & 0 \ldots 0 \\
0 \ldots 0 & 0 \ldots 0 & 0 \ldots 0
\end{array}\right)\left(\begin{array}{c}
w_{0} \\
\vdots \\
w_{n} \\
s_{0} \\
\vdots \\
s_{n} \\
z_{0} \\
\vdots \\
z_{n}
\end{array}\right)
$$

where, $\overline{\mathbf{L}}_{\mathbf{1}}, \overline{\mathbf{L}}_{\mathbf{2}}, \overline{\mathbf{0}}$ and $\overline{\mathbf{I}}$ are the sub matrices of $\mathbf{L}_{\mathbf{1}}, \mathbf{L}_{\mathbf{2}}, \mathbf{0}$ and $\mathbf{I}$ respectively. Now we have generalized eigen value (4.30), we can find the minimum eigen values $R(a)$, then the critical Rayleigh number values $R_{c}$ and the corresponding wave number $a_{c}$ for various values of $Q$ using MATLAB software, the results are illustrated in the next section.

\section{Results And Conclusion}

- Spectral Chebyshev Tau method give results in full agreement with the results that obtained by the analytical solution given by Chandrasekhar [1].

- For the three cases of the boundaries free-free, rigid-rigid and rigid-free the critical Rayleigh number determine the stability. if $R<R_{c}$ the motion is stable and no convection, when $R=R_{c}$ it is stationary convection, and unstable motion when $R>R_{c}$.

- It is also observed the effect of the magnetic field, as $Q$ increases, the value of the critical Rayleigh number and the critical wave number also increases. 


\begin{tabular}{|c|c|c|c|c|}
\hline \multirow{2}{*}{$Q$} & \multicolumn{2}{|c|}{ Chandrasekhar [1] } & \multicolumn{2}{c|}{ Present study } \\
& $a_{c}$ & $R_{c}$ & $a_{c}$ & $R_{c}$ \\
\hline 0 & 2.233 & 657.511 & 2.22 & 657.51 \\
\hline 10 & 2.590 & 923.070 & 2.59 & 923.07 \\
\hline 50 & 3.270 & 1762.04 & 3.27 & 1762.04 \\
\hline 100 & 3.702 & 2653.71 & 3.7 & 2653.71 \\
\hline 200 & 4.210 & 4258.49 & 4.21 & 4258.49 \\
\hline 500 & 4.998 & 8578.88 & 5.00 & 8578.89 \\
\hline 1000 & 5.684 & 15207.0 & 5.68 & 15207 \\
\hline 2000 & 6.453 & 27699.9 & 6.46 & 27699.79 \\
\hline 5000 & 7.585 & 63135.9 & 7.61 & 63135.48 \\
\hline 10000 & 8.588 & 119832 & 8.61 & 119831.71 \\
\hline 20000 & 9.706 & 230038 & 9.72 & 230038.48 \\
\hline 40000 & 10.95 & 445507 & 10.96 & 445506.86 \\
\hline
\end{tabular}

TABLE 1. The relation between between $R_{c}$ and $a_{c}$ when the boundaries are free-free and $Q=0,10,50, \ldots, 40000$.

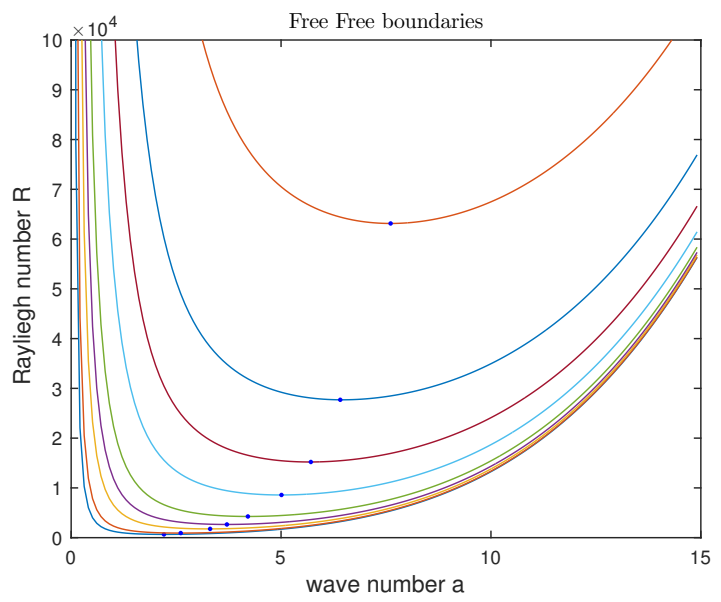

Figure 1. The relation between between $R_{c}$ and $a_{c}$ when the boundaries are free-free and $Q=0,10,50, \ldots, 40000$. 


\begin{tabular}{|c|c|c|c|c|}
\hline \multirow{2}{*}{$Q$} & \multicolumn{2}{|c|}{ Chandrasekhar [1] } & \multicolumn{2}{c|}{ Present study } \\
& $a_{c}$ & $R_{c}$ & $a_{c}$ & $R_{c}$ \\
\hline 0 & 3.13 & 1707.8 & 3.12 & 1707.77 \\
\hline 10 & 3.25 & 1945.9 & 3.27 & 1945.75 \\
\hline 50 & 3.68 & 2803.1 & 3.68 & 2802.01 \\
\hline 100 & 4.00 & 3757.4 & 4.01 & 3757.23 \\
\hline 200 & 4.45 & 5488.6 & 4.45 & 5488.54 \\
\hline 500 & 5.16 & 10110 & 5.16 & 10109.78 \\
\hline 1000 & 5.80 & 17103 & 5.81 & 17102.85 \\
\hline 2000 & 6.55 & 30125 & 6.56 & 30124.81 \\
\hline 4000 & 7.40 & 54697 & 7.39 & 54700.75 \\
\hline 6000 & 7.94 & 78391 & 7.93 & 78441.25 \\
\hline 8000 & 8.34 & 101606 & 8.30 & 101930.17 \\
\hline 10000 & 8.66 & 124509 & 8.52 & 125856.50 \\
\hline
\end{tabular}

TABLE 2. The relation between between $R_{c}$ and $a_{c}$ when the boundaries are rigid-rigid and $Q=0,10,50, \ldots, 10000$.

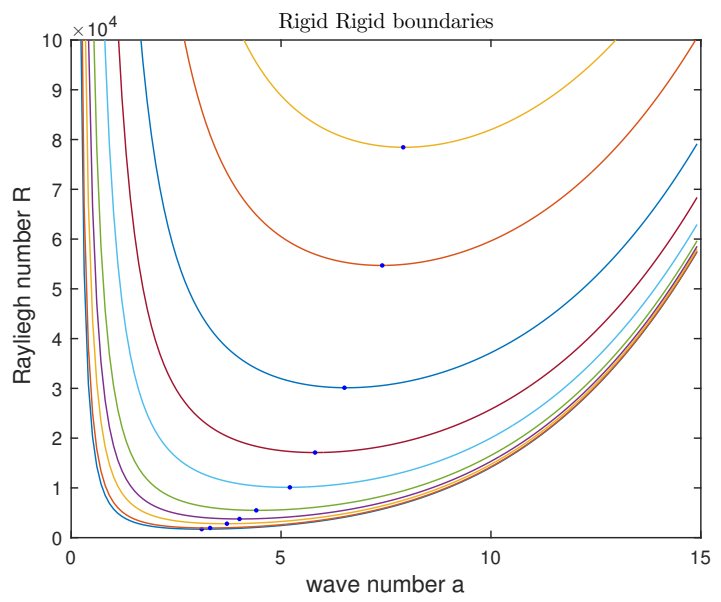

Figure 2. The relation between between $R_{c}$ and $a_{c}$ when the boundaries are rigid-rigid and $Q=0,10, \ldots, 10000$. 


\begin{tabular}{|c|c|c|c|c|}
\hline \multirow{2}{*}{$Q$} & \multicolumn{2}{|c|}{ Chandrasekhar [1] } & \multicolumn{2}{c|}{ Present study } \\
& $a_{c}$ & $R_{c}$ & $a_{c}$ & $R_{c}$ \\
\hline 0 & 2.68 & 1100.75 & 2.7 & 1100.81 \\
\hline 2.5 & 2.75 & 1167.2 & 2.71 & 1167.45 \\
\hline 12.5 & 2.97 & 1415.5 & 3.01 & 1415.81 \\
\hline 25 & 3.17 & 1699.4 & 3.21 & 1699.79 \\
\hline 50 & 3.45 & 2217.6 & 3.51 & 2217.98 \\
\hline 125 & 4.00 & 3586.1 & 4.01 & 3585.85 \\
\hline 250 & 4.50 & 5613.3 & 4.51 & 5612.93 \\
\hline 500 & 5.10 & 9304.5 & 5.11 & 9303.95 \\
\hline 1000 & 5.75 & 16119 & 5.71 & 16118.68 \\
\hline 1500 & 6.20 & 22592 & 6.21 & 22591.30 \\
\hline 2000 & 6.50 & 28879 & 6.51 & 28877.86 \\
\hline 2500 & 6.75 & 35044 & 6.81 & 35043.57 \\
\hline 5000 & 7.65 & 64847 & 7.61 & 64836.70 \\
\hline 10000 & 8.65 & 122140 & 8.71 & 121512.72 \\
\hline
\end{tabular}

TABLE 3. The relation between between $R_{c}$ and $a_{c}$ when the boundaries are rigid-free and $Q=0,2.5,12.5, \ldots, 10000$.

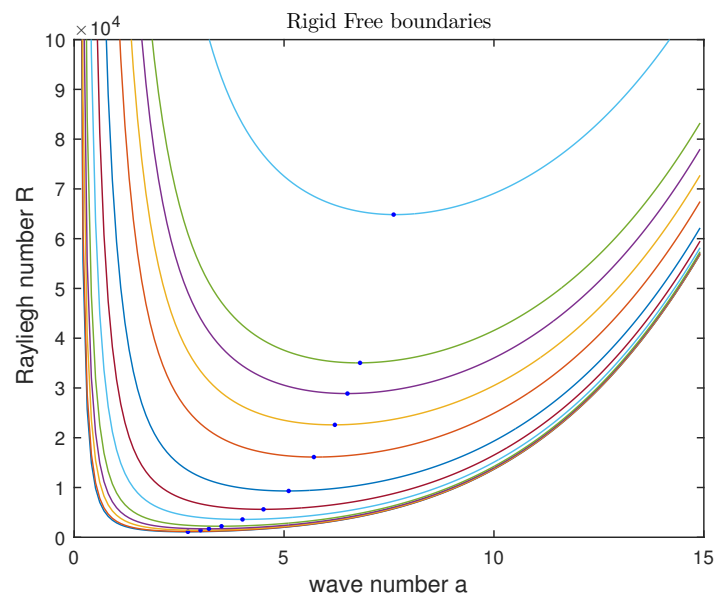

Figure 3. The relation between between $R_{c}$ and $a_{c}$ when the boundaries are rigid-free and $Q=0,2.5,12.5 \ldots, 10000$. 
Conflicts of Interest: The author(s) declare that there are no conflicts of interest regarding the publication of this paper.

\section{REFERENCES}

[1] S. Chandrasekhar, Hydrodynamic and hydromagnetic stability, Oxford University Press, Oxford, 1961.

[2] S. Chandrasekhar, The instability of a layer of fluid heated below and subject to Coriolis forces, Proc. R. Soc. Lond. A. 217 (1953), 306-327.

[3] S. Chandrasekhar, The instability of a layer of fluid heated below and subject to the simultaneous action of a magnetic field and rotation, Proc. R. Soc. Lond. A. 225 (1954), 173-184.

[4] S. Chandrasekhar, D.D. Elbert, The instability of a layer of fluid heated below and subject to Coriolis forces. II, Proc. R. Soc. Lond. A. 231 (1955), 198-210.

[5] A.J. Chorin, A numerical method for solving incompressible viscous flow problems, J. Comput. Phys. 2 (1967), $12-26$.

[6] J.J. Dongarra, B. Straughan, D.W. Walker, Chebyshev tau-QZ algorithm methods for calculating spectra of hydrodynamic stability problems, Appl. Numer. Math. 22 (1996), 399-434.

[7] L. Fox, Chebyshev Methods for Ordinary Differential Equations, Computer J. 4 (1962), 318-331.

[8] M.A. Hernandez, Chebyshev's approximation algorithms and applications, Comput. Math. Appl. 41 (2001), $433-445$.

[9] A.A. Hill, Convection in porous media and Legendre, Chebyshev Galerkin methods, Ph.D. Thesis, Durham University, 2005.

[10] D. Johnson, Chebyshev Polynomials in the Spectral Tau Method and Applications to Eigenvalue Problems, NASA Contractor Report 198451, 1996.

[11] S. Talukdar, A study of some steady and unsteady MHD flow problems with heat and mass transfer, Ph.D. Thesis, Gauhati University, 2013.

[12] D.Y. Tzou, Instability of Nanofluids in Natural Convection, J. Heat Transfer, 130 (2008), 072401.

[13] B.-F. Wang, D.-J. Ma, Z.-W. Guo, D.-J. Sun, Linear instability analysis of Rayleigh-Bénard convection in a cylinder with traveling magnetic field, J. Crystal Growth, 400 (2014), 49-53.

[14] D. Yadav, J. Wang, R. Bhargava, J. Lee, H.H. Cho, Numerical investigation of the effect of magnetic field on the onset of nanofluid convection, Appl. Therm. Eng. 103 (2016), 1441-1449. 